\title{
Osteogenic response of mesenchymal stem cells to continuous mechanical strain is dependent on ERK1/2-Runx2 signaling
}

\author{
PENG ZHANG $^{1}$, YUQIONG WU ${ }^{1}$, ZONGLAI JIANG ${ }^{2}$, LINGYONG JIANG $^{1}$ and BING FANG ${ }^{1}$ \\ ${ }^{1}$ Center of Craniofacial Orthodontics, Department of Oral and Maxillofacial Surgery, Ninth People's Hospital, \\ Shanghai Jiao Tong University School of Medicine, Shanghai Key Laboratory of Stomatology, Shanghai 200011; \\ ${ }^{2}$ Institute of Mechanobiology and Medical Engineering, Shanghai Jiao Tong University, Shanghai 200240, P.R. China
}

Received December 24, 2011; Accepted February 9, 2012

DOI: $10.3892 / \mathrm{ijmm} .2012 .934$

\begin{abstract}
Mechanical stimuli are responsible for bone remodeling during orthodontic tooth movement. The role of mechanical stimulation in the regulation of the fate of bone mesenchymal stem cells (BMSCs) is of interest in bone regeneration and tissue engineering applications. However, the signaling pathway involved in strain-induced biochemical events in BMSCs is not well established and can be controversial. This study investigated strain-induced proliferation and differentiation of BMSCs, as well as the mechanism of mechanotransduction. BMSCs were exposed to continuous mechanical strain (CMS) of $10 \%$ at $1 \mathrm{~Hz}$. The results showed that CMS reduced the proliferation of BMSCs and stimulated osteogenic differentiation by activating Runx2, followed by increased alkaline phosphatase (ALP) activity and mRNA expression of osteogenesis-related genes (ALP, collagen type I and osteocalcin). Furthermore, the phosphorylation level of extracellular regulated protein kinase (ERK)1/2 increased significantly at the onset of strain. However, the presence of U0126, a selective inhibitor of ERK1/2, blocked the induction of Runx 2 and subsequent osteogenic events. These findings demonstrate that CMS regulated Runx2 activation and favored osteoblast differentiation through activation of the ERK1/2 signaling pathway. These results will contribute to a better understanding of strain-induced bone remodeling and will form the basis for the correct choice of applied force in orthodontic treatment.
\end{abstract}

\section{Introduction}

Mechanical loading is known to be an important factor in regulating the formation and renewal of bone in many biological

Correspondence to: Dr Lingyong Jiang or Dr Bing Fang, Center of Craniofacial Orthodontics, Department of Oral and Maxillofacial Surgery, Ninth People's Hospital, Shanghai Jiao Tong University School of Medicine, Shanghai Key Laboratory of Stomatology, No. 639 Zhi-Zao-Ju Road, Shanghai 200011, P.R. China

E-mail: jly117@sina.com

E-mail: fangbing@sjtu.edu.cn

Key words: bone mesenchymal stem cell, continuous mechanical strain, extracellular regulated protein kinase 1/2-Runx2, osteogenesis events, such as orthodontic tooth movement. Orthodontic forces create both compression and tension sites in the periodontal ligament, causing bone synthesis and resorption, respectively, and ultimately tooth movement takes place $(1,2)$. A number of studies have proved that different types of loading have different effects on bone cells. These effects depend on the magnitude, duration, frequency and mode of the strain (3-5).

Recent evidence has indicated that the subsequent activation of bone mesenchymal stem cells (BMSCs) in response to orthodontic force may play a key role in the whole process (6). BMSCs are force-sensitive cells and are capable of detecting, transducing and responding to an extracellular stimulus, with alteration in their biological characteristics (7). Force-subjected BMSCs in periodontal ligament may differentiate into osteoblasts and at the same time osteoclasts, thus regulating bone regeneration and expected tooth movement. Nevertheless, the specific mechanobiological mechanism of orthodontic tooth movement has not been thoroughly studied.

The activation of specific transcription factors is essential for cellular commitment to a differentiation lineage. To date, many experiments have demonstrated the important role of Runx 2 in regulating osteogenic differentiation (8). As a component of the runt family of transcription factors, Runx2 is suggested to be the key in different signaling pathways involved in mechanotransduction $(9,10)$. Runx 2 can induce the synthesis of alkaline phosphatase (ALP), collagen type I (COLI) and osteocalcin (OC) (11), as well as the maturation of osteoblast phenotype.

Experimental evidence suggests that the mitogen-activated protein kinase (MAPK) pathway is essential at the beginning of osteoblast differentiation (12). Critical members of the MAPKs include extracellular regulated protein kinase (ERK)1/2, which is an essential molecule for mechanotransduction (13). Runx 2 can be phosphorylated and activated by ERK1/2 pathways (14). However, the distinct signal transduction pathway that links mechanical strain with the regulation of gene expression is not clear.

Our aim is to investigate the initial osteogenic effect of continuous mechanical strain on rat BMSCs and the key mechanism associated with osteodifferentiation of MSCs. In this study, we used the FX-4000T ${ }^{\mathrm{TM}}$ Tension Plus ${ }^{\mathrm{TM}}$ unit to mimic a mechanical environment which cells were subjected to at the CMS-induced tension site. The osteogenesis process was 
evaluated by the ALP activity assay, and the mRNA expression of osteogenic markers such as Runx2, ALP, COLI and OC. The function of ERK1/2 in mechanotransduction was studied by investigating the expression of phosphorylated ERK1/2 during CMS. That ERK1/2 functioned on Runx2 was examined by inhibition of ERK1/2 activity with U0126 pre-treatment.

\section{Materials and methods}

Harvest and culture of rat BMSCs. Rat BMSCs were isolated from the femurs and tibia of 3-month-old Sprague-Dawley rats. The cells were supplemented in $\alpha$-modified Eagle's culture medium (HyClone) with $10 \%$ fetal bovine serum (FBS) (Invitrogen) and antibiotics (100 U/ml penicillin G and $100 \mu \mathrm{g} / \mathrm{ml}$ streptomycin sulfate), and finally incubated at $37^{\circ} \mathrm{C}$ in a humidified incubator with $95 \%$ air and $5 \% \mathrm{CO}_{2}$. The medium was refreshed every 3 days. After reaching 70-80\% confluence, cells were subcultured or plated for subsequent experiments. BMSCs were identified as CD44(+), CD54(+), and CD34(-); furthermore, BMSCs cultured in adipogenic or osteogenic media differentiated into adipocytes and osteoblasts, respectively (data not shown). BMSCs between passages 3-6 were used in our experiments.

Application of continuous mechanical strain. BMSCs were seeded on Flexercell 6-well silicone rubber plates at a density of $2 \times 10^{4} / \mathrm{cm}^{2}$. The cells were cultured for $24-48 \mathrm{~h}$, allowing them to attach and reach $80 \%$ confluence. In this study, cells were subjected to mechanical strain of $10 \%$, elongation at $1 \mathrm{~Hz}$ sinusoidal curve for $96 \mathrm{~h}$, using the FX-4000T ${ }^{\mathrm{TM}}$ Flexercell Tension Plus ${ }^{\mathrm{TM}}$ unit (Flexercell International Corporation). Control cells were cultured on the same plates and incubated under the same conditions for the maximum stretching period.

MTT assay for cell proliferation. Cells with or without CMS for 24 and $48 \mathrm{~h}$, were detached from the elastic silicone film and seeded on 96-well plates at a density of $1 \times 10^{4} /$ well. At $6 \mathrm{~h}$, a $20 \mu \mathrm{l}$ sample of MTT solution (5 g/l dissolved in PBS, Sino-American) was added to the 96-well plate and incubated at $37^{\circ} \mathrm{C}$ for $4 \mathrm{~h}$. The supernatant was then removed and $150 \mu \mathrm{l}$ dimethylsulfoxide was added. After vibration to fully dissolve MTT formazan, the absorbance of samples was measured using a spectrophotometer at a wavelength of $490 \mathrm{~nm}$ (BioRad). Pure culture medium without BMSCs was used as the blank control. The number of viable cells was represented as the relative ratio of their absorbance to that of the blank.

Quantification assay of ALP activity. ALP activity of the cell lysate was measured using p-nitrophenylphosphate (pNPP), as described previously (15). ALP activity was expressed as nmol p-nitrophenol produced per min/mg of total protein. Data were presented as fold changes over the non-loaded group at the respective time points.

ALP staining. The cultured cells were rinsed with pre-cooled PBS three times and fixed with $4 \%$ paraformaldehyde for $10 \mathrm{~min}$ at room temperature. Coloration was then assessed according to the manufacturer's instructions using the BCIP/ NBT Alkaline Phosphatase Color Development kit (Beyotime Institute of Biotechnology).

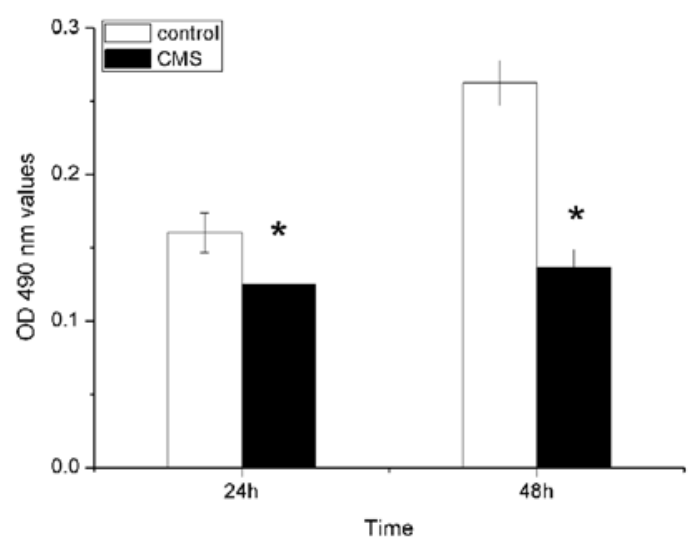

Figure 1. The effect of CMS on the proliferation of BMSCs. Total cell proliferation was determined at 24 and $48 \mathrm{~h}$ with or without CMS. Data were combined from three individual experiments and shown as mean $\pm \mathrm{SD}$. There are significant differences at ${ }^{*} \mathrm{P}<0.05$.

Real-time RT-PCR analysis. After mechanical loading, cells were washed twice with ice-cold PBS. Total-RNA was extracted using TRIzol reagent (Invitrogen) and cDNA was reverse transcribed from mRNA with a RevertAid ${ }^{\mathrm{TM}}$ First Strand cDNA Synthesis kit (Fermentas) according to the manufacturer's instructions. Real-time PCR reaction was monitored by an ABI 7900 HT system using SYBR ${ }^{\circledR}$ Premix Ex Taq ${ }^{\text {TM }}$ (Takara Bio, Inc.). The settings were as follows: denaturation at $95^{\circ} \mathrm{C}$ for $10 \mathrm{sec}$, and then 40 cycles $\left(10 \mathrm{sec}\right.$ at $95^{\circ} \mathrm{C}, 30 \mathrm{sec}$ at $\left.60^{\circ} \mathrm{C}\right)$. A dissociation curve was constructed to confirm that there was no non-specific amplification. mRNA expression of relevant genes were normalized to that of $\beta$-actin as a house-keeping gene, respectively. The primer sequences used in this study were as follows: $\beta$-actin, forward, 5'-CACCCGCGAGTACAACCT TC-3' and reverse, 5'-CCCATACCCACCATCACACC-3'; ALP, forward, 5'-TATGTCTGGAACCGCACTGAAC-3' and reverse, 5'-CACTAGCAAGAAGAAGCCTTTGG-3'; COLI, forward, 5'-CAGGCTGGTGTGATGGGATT-3' and reverse, 5'-CCAAG GTCTCCAGGAACACC-3'; OC, forward, 5'-GCCCTGACT GCATTCTGCCTCT-3' and reverse, 5'-TCACCACCTTACT GCCCTCCTG-3'; Runx2, forward, 5'-ATCCAGCCACCTT CACTTACACC-3' and reverse, 5'-GGGACCATTGGGAACT GATAGG-3'.

Analysis of protein level by western blotting. An equal amount of protein extract per sample was subjected to $10 \%$ SDS-PAGE and electro-transferred to nitrocellulose membranes. After blocking, membranes were probed with primary antibodies, followed by incubation with the ALP-linked IgG secondary antibody (1:500, Jackson Immunoresearch). The protein bands were visualized by NBT/BCIP substrates. The primary antibodies used in our experiment were as follows: anti-Runx2 (mouse monoclonal anti-Runx2, 1:500, Abcam); anti-ERK1/2 (rabbit monoclonal anti-p44/42 MAPK, 1:500, Cell Signaling Technology); anti-phospho-ERK1/2 [rabbit monoclonal anti-phospho-p44/42 MAPK (Thr 202/Tyr 204), 1:500, Cell Signaling Technology]. For normalization of protein levels, anti- $\alpha$-tubulin (1:500, Santa Cruz Biotechnology, Inc.) was used. Runx 2 levels were normalized to $\alpha$-tubulin expression, and phospho-ERK1/2 to their respective total protein levels. 
A

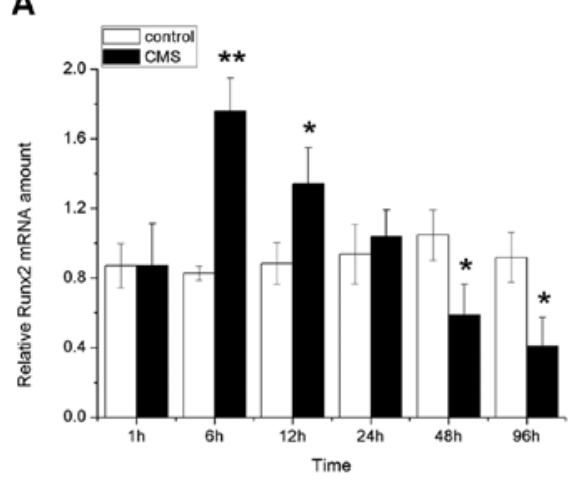

C

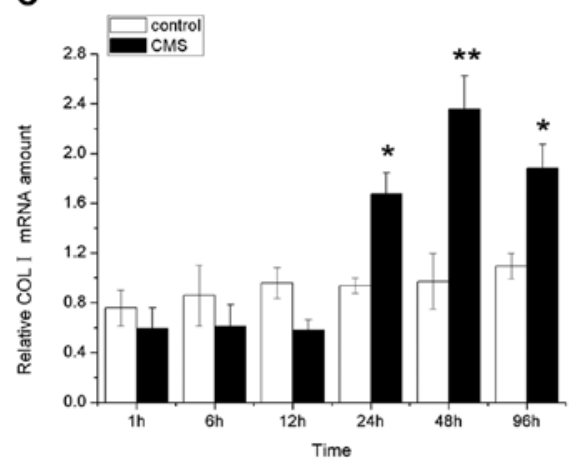

E

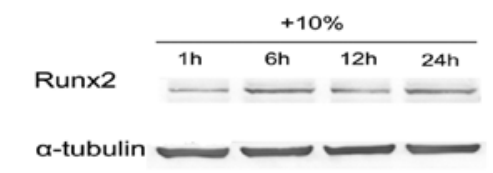

$\mathbf{F}$

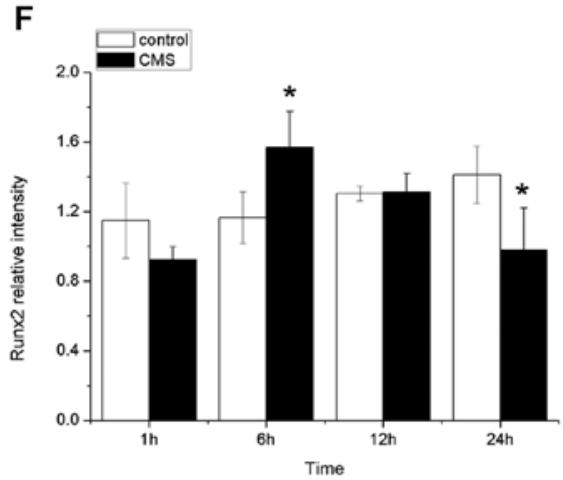

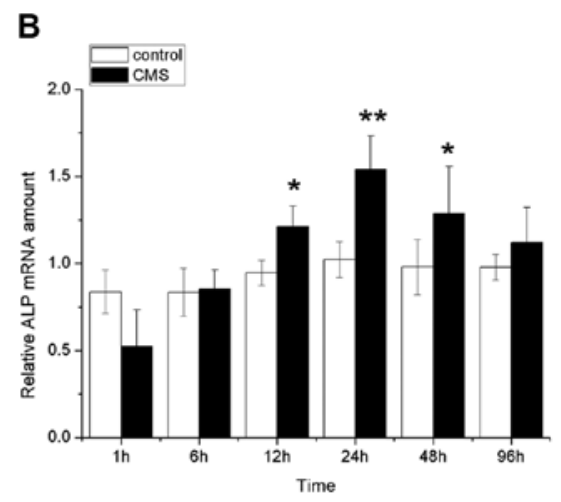

D
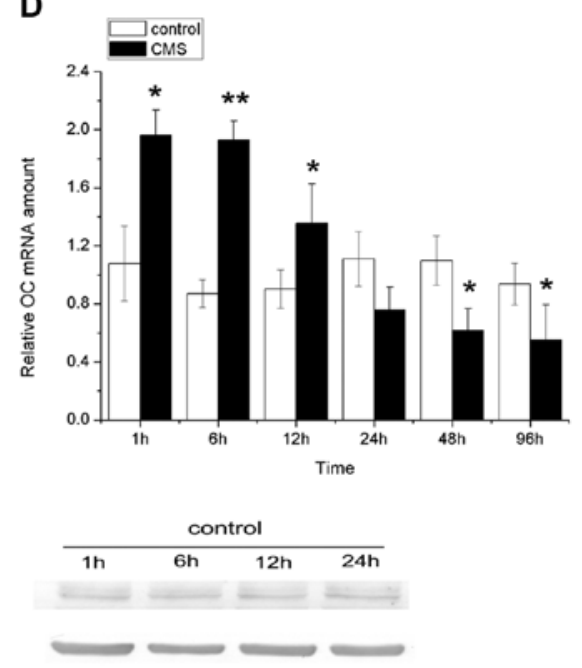

$\mathbf{G}$

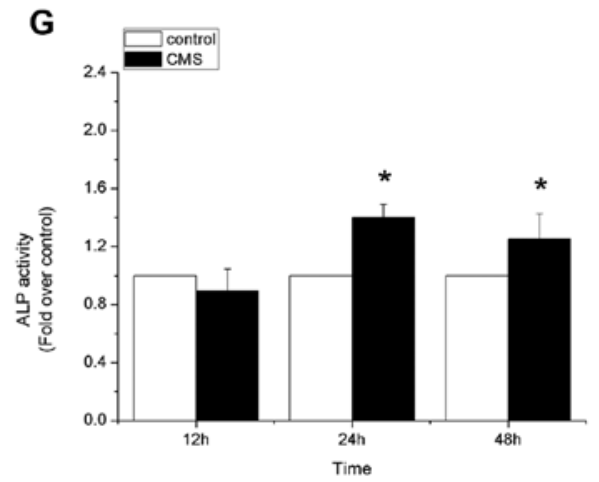

Figure 2. Osteoblastic differentiation through Runx2 in mechanically strained BMSCs. The mRNA changes of specific osteoblastic genes at different time points (0-96 h) following CMS treatment are showed in (A-D). (A) Runx2, (B) ALP, (C) COLI, (D) OC, (E and F) show the changes of Runx2 protein level at different time points (0-24 h) after CMS. Runx2 protein levels are normalized to $\alpha$-tubulin levels. ALP activities of BMSCs for $48 \mathrm{~h}$ CMS are assessed in (G). Control samples without CMS were prepared at the same time points. Each experiment was performed three times individually and similar results were obtained each time. Results are presented as the mean \pm SD. Significant differences among each group are noted by ${ }^{*} \mathrm{P}<0.05$ and ${ }^{* * *} \mathrm{P}<0.01$.

RNA interference studies. The target mRNA sequence of Runx2-rattus (NM_053470.1) was searched in NCBI GenBank. Small interfering RNAs (siRNA) against rat Runx2 were designed and synthesized by GenePharma Biological Company (Shanghai, China). SiRNA which did not recognize any known homology of rattus genes was used as a negative control. After seeding on the Flexercell plate and reaching approximately $80 \%$ confluence, BMSCs were transfected with siRNA using Lipofectamine (Invitrogen) according to the manufacturer's protocol. The cells were then cultured for another $24 \mathrm{~h}$ before mechanical loading. Negative control (NC) groups were transfected with non-silencing siRNA instead of target siRNA.
Inhibition of ERK1/2 signal transduction pathway. In order to assess the role of the ERK1/2 signaling pathway in the straininduced differentiation of BMSCs, the selective inhibitor U0126 (Cell Signaling Technology) was used. Preliminary experiments showed that the optimal concentration of U0126 was $10 \mathrm{mM}$. Cells were pre-treated with U0126 for $1 \mathrm{~h}$ prior to the strain stimulus, and U0126 was present during the whole strain experiment. Mechanically stretched cells pre-treated with dimethylsulfoxide (DMSO) were used as a control group.

Statistical analysis. Each experiment was performed a minimum of three times. The data were expressed as mean \pm SD. Significant 
differences were determined using Student's t-test. A value of $\mathrm{P}<0.05$ was considered statistically significant.

\section{Results}

The role of CMS in the proliferation of BMSCs. Compared to controls, CMS caused a significant change in proliferation. CMS decreased the proliferation of cells in the experimental group, and this trend was obvious with elongation of loading time (Fig. 1).

Induction of osteogenesis through Runx2 by CMS. To determine the osteogenic commitment of BMSCs, we examined the mRNA expression of certain osteogenic-related markers including Runx2, ALP, COLI and OC. Runx 2 mRNA level in the strain group showed a sharp increase at $6 \mathrm{~h}$, with a 2-fold increase compared to the control group (Fig. 2A), but decreased with elongation of loading. The levels of ALP and COLI were found to be upregulated later than Runx2, which reached its peak at 24 or $48 \mathrm{~h}$ (Fig. 2B and C). OC mRNA expression showed a significant increase at the onset of strain, but diminished quickly (Fig. 2D). We then examined whether the upregulation of Runx2 mRNA was in accordance with its protein expression. Although not upregulated within $1 \mathrm{~h}$, a sharp increase in Runx 2 protein was observed at $6 \mathrm{~h}$ and remained elevated at $12 \mathrm{~h}$ (Fig. 2E and F). To confirm early improved osteogenesis, we also tested ALP activity and its straining. In agreement with the above results, BMSCs subjected to CMS showed higher ALP activities (Fig. 2G) and deeper straining at $24 \mathrm{~h}$ (Fig. 3A and B).

Since Runx2 plays an important role in osteoblast differentiation, we then investigated its function in strain-induced osteogenesis. We transfected BMSCs with Runx2-siRNA, and examined the effect of Runx2 downregulation (Fig. 4A). Following Runx2-target siRNA treatment for 6 and $24 \mathrm{~h}$, determination of BMSCs osteogenic capacity showed that Runx2 knockdown clearly inhibited the CMS-induced activation of marker genes (Fig. 4B-D). In addition, the decreased expression of osteogenic markers was associated with lighter ALP straining in the siRNA treated group after $24 \mathrm{~h}$ (Fig. 3C and D).

To confirm that CMS could induce BMSCs into an osteoblastic lineage, we prolonged the loading time to $96 \mathrm{~h}$ and observed the mRNA expression of specific markers. The results showed that the levels of all detected genes returned to control levels or lower within 96 h (Fig. 2A-D).

Induction of ERK1/2 pathway by CMS. The data showed that ERK1/2 was significantly phosphorylated within a short period after the onset of stimuli, reaching its maximum level at 30 min (Fig. 5). The phosphorylation level then dropped gradually but still remained higher than the unstrained group after $2 \mathrm{~h}$. After $6 \mathrm{~h}$ loading, the level of phosphorylated ERK1/2 almost returned to baseline and was lower than the unstrained group.

Induction of osteogenesis through the ERK1/2-Runx2 pathway by CMS. To address whether the accumulation of ERK1/2 was paralleled by CMS-induced osteogenesis, we assessed the expression of osteogenic-related genes in the presence of
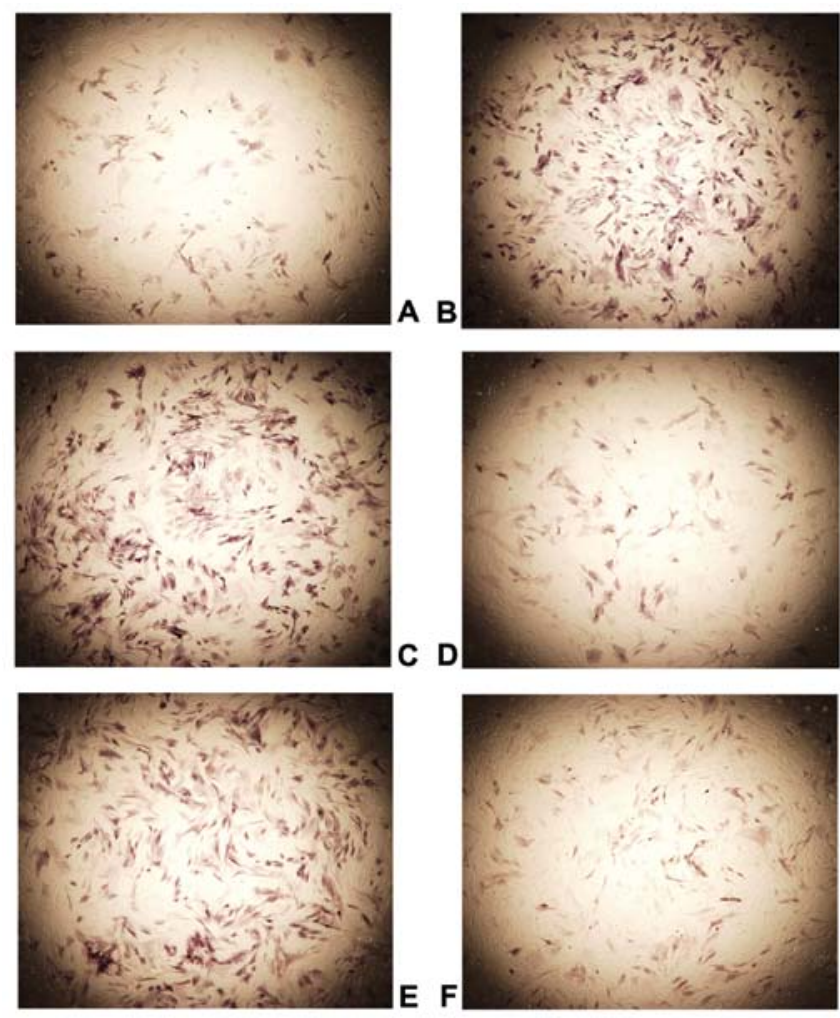

Figure 3. ALP staining at $24 \mathrm{~h}$ in different conditions. (A) Control group, (B) CMS group, (C) CMS with non-silencing siRNA, (D) CMS with Runx2target siRNA, (E) CMS plus DMSO, (F) CMS plus ERK1/2 selective inhibitor U0126. The experiments were performed three times; similar results were obtained each time (x100).

U0126, a potent and selective inhibitor of ERK1/2 kinase. A MTT viability assay demonstrated that the inhibitor had no cytotoxic effects on cells (data not shown). Pre-treatment with U0126 significantly blocked the strain-induced activation of ERK1/2 (Fig. 5). In addition, a significant decrease in the protein level of Runx2 was observed (Fig. 6A), with down-regulated levels of ALP, COLI and OC mRNA in strained cells after $24 \mathrm{~h}$ (Fig. 6B-D). Similarly, U0126 pretreatment decreased strain-stimulated ALP activity at $24 \mathrm{~h}$ (Fig. 3E and F), suggesting that the ERK1/2-Runx2 pathway was involved in the transduction of external strain signals.

\section{Discussion}

It is well known that mechanical loading is a fundamental determinant of bone formation and reconstruction. It can be converted into a cellular response that involves rapid, kinasemediated changes in gene expression (16). Physiological strains reported for daily activities in human long bones are of the order of 2,000-4,000 $\mu$ strain (17). Orthodontic tooth movement induced by mechanical stimuli is dependent upon the remodeling capacity of local alveolar bone. Force may cause bone resorption and apposition in the pressure and tension sites, respectively. Recently, more attention has been placed on the diverse responses of bone cells $(18,19)$. In this study, the magnitude of $10 \%(10,000 \mu$ strain) was chosen according to stress analysis of the periodontal ligament under various orthodontic loadings (20). 

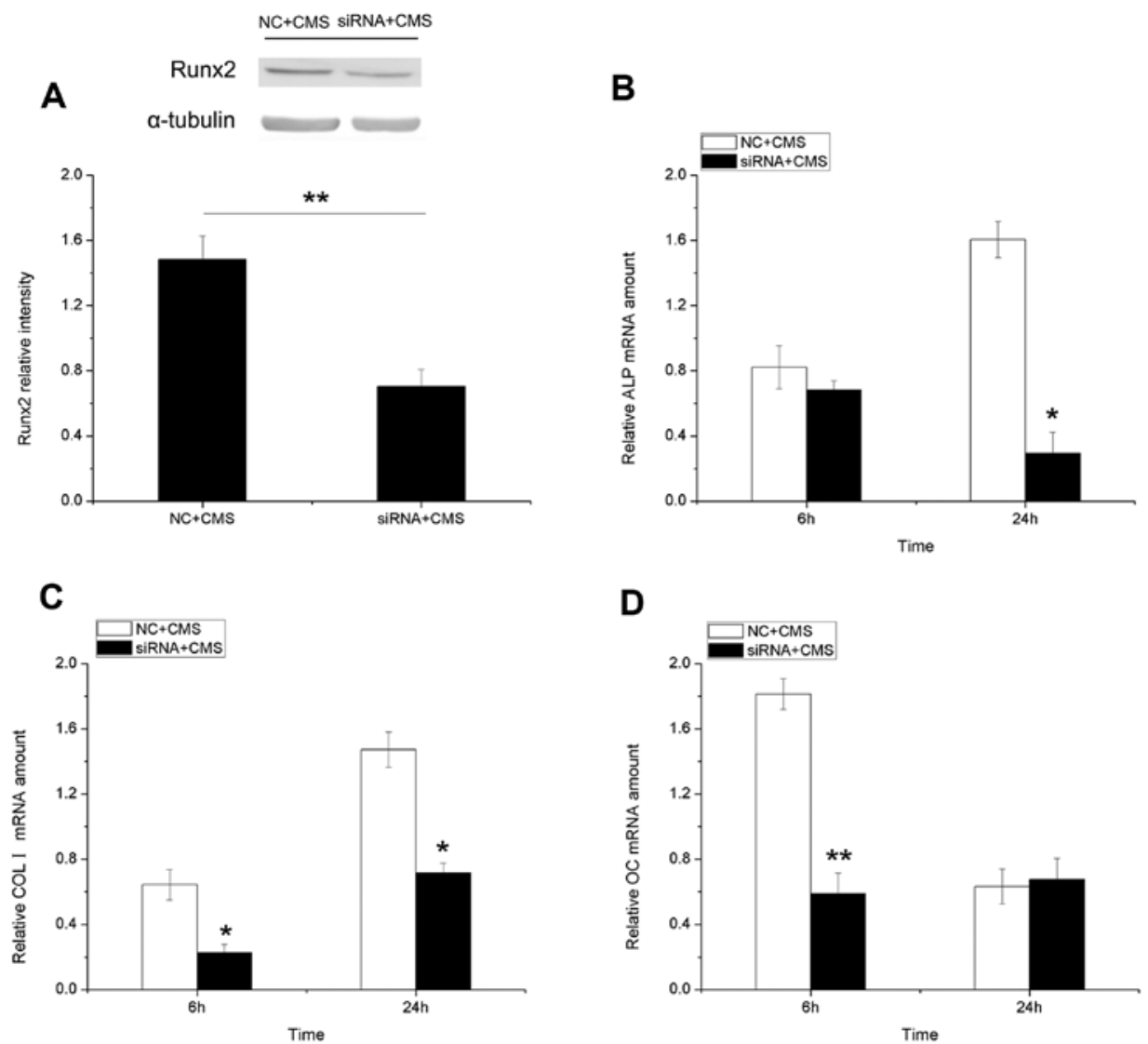

Figure 4. Effect of Runx2 interfering on the osteogenesis-related genes in mechanically stretched BMSCs. (A) Runx2 knockdown was determined by western blotting in non-silencing siRNA-treated and Runx 2 siRNA-treated group after $6 \mathrm{~h} \mathrm{CMS.} \mathrm{mRNA} \mathrm{levels} \mathrm{of} \mathrm{ALP,} \mathrm{COLI} \mathrm{and} \mathrm{OC} \mathrm{at} \mathrm{indicated} \mathrm{time} \mathrm{points} \mathrm{(6} \mathrm{and} 24 \mathrm{~h}$ ) were displayed (B-D). Values were combined from three individual experiments and shown as mean $\pm \mathrm{SD}(\mathrm{n}=3)$. There are significant differences at ${ }^{*} \mathrm{P}<0.05$ and ${ }^{* *} \mathrm{P}<0.01$.
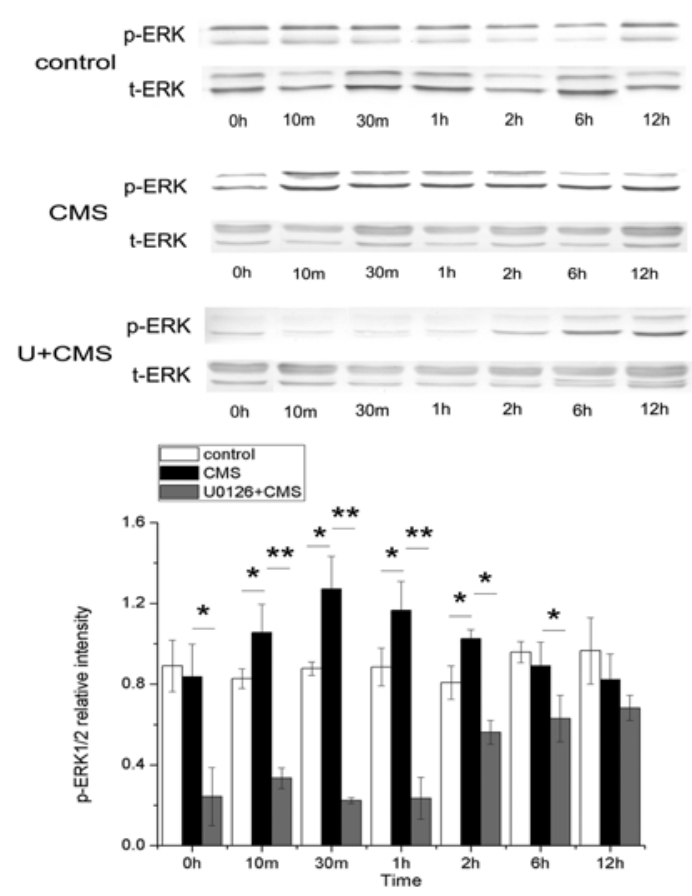

Figure 5. ERK1/2 signaling pathway involved in CMS-induced osteoblastic differentiation. Phosphorylation of ERK1/2 protein levels were examined by western blotting after exposure to CMS or U0126 pre-treatment at the indicated time point $(0-12 \mathrm{~h})$. Control samples without CMS were prepared at the same time points. Phospho-ERK1/2 levels are normalized to their respective total protein levels. The image is representative of three separate experiments. Values are mean $\pm S D(n=3)$. There are significant differences at ${ }^{*} \mathrm{P}<0.05$ and ${ }^{* *} \mathrm{P}<0.01$.
The effect of mechanical loading on the proliferation of osteoblastic cells is controversial. Some studies have shown that an appropriate mechanical force can induce the growth of BMSCs (21), while others have shown the opposite findings (22). Our findings suggested that the proliferation of BMSCs was suppressed by CMS, and this response was sensitive to stimuli duration. When considering the balance between BMSCs proliferation and their tissue-specific differentiation, the cessation of cell proliferation may mark the onset of osteogenic lineage commitment.

Runx2 is a key regulator which plays an important role in mastering the transcription of many genes involved in osteogenic differentiation, including ALP, COLI, OC and osteopontin (23). Our data were consistent with previous reports stating that mechanical stress induced the expression of the osteogenic transcription factor Runx 2 at mRNA and/or protein levels (24). The expression of ALP, COLI and OC were elevated following the early application of CMS. These results were in agreement with several reports which suggested that mechanical strain could enhance osteogenesis via up-regulation of several osteoblastic genes under the regulation of Runx2 (25). When treated with Runx2-target siRNA, certain osteogenic-related genes were significantly decreased. These data demonstrated that BMSCs underwent an early osteoblastic lineage, and Runx 2 was the predominant activator responsible for this differentiation.

It should be noted that the levels of all detected genes returned to control levels or lower within $96 \mathrm{~h}$. This could 

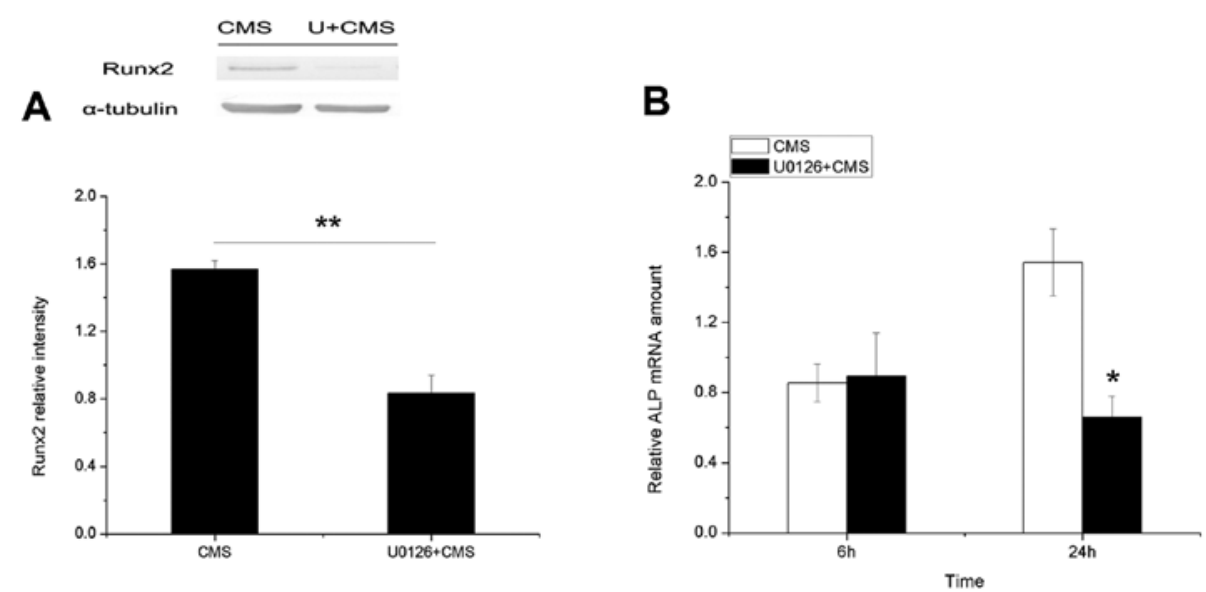

C

D
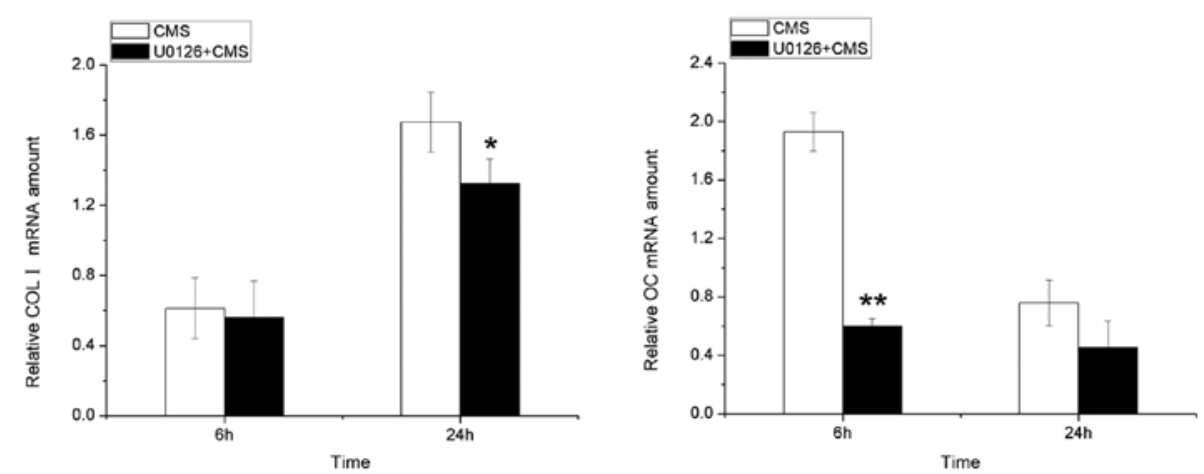

Figure 6. Effects of ERK1/2 selective inhibitor (U0126) on mechanical strain-induced osteoblastic differentiation of BMSCs through Runx2 activation. (A) Protein levels of Runx 2 treated with U0126 after $6 \mathrm{~h}$ CMS were visualized by western blotting. mRNA levels of (B) ALP, (C) COLI and (D) OC at 6 and $24 \mathrm{~h}$ with U0126 were quantified by real-time RT-PCR. Data were combined from three individual experiments and shown as mean \pm SD. There are significant differences at ${ }^{*} \mathrm{P}<0.05$ and ${ }^{* *} \mathrm{P}<0.01$.

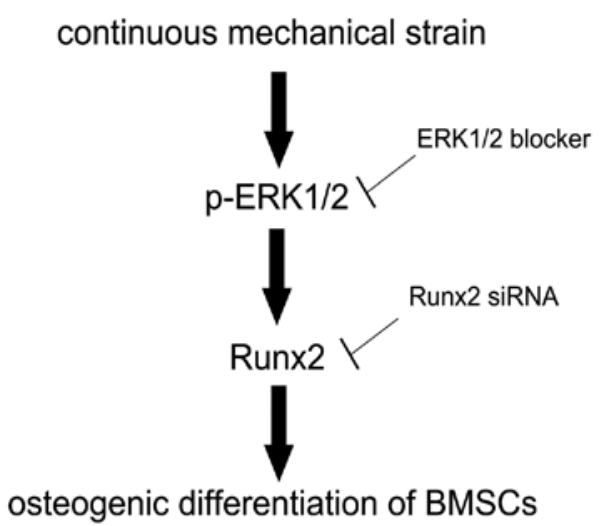

Figure 7. Schematic drawing outlining the ERK1/2-Runx2 pathway that mediates the osteoblastic differentiation of BMSCs by CMS in this study.

indicate that the CMS employed in our study may not totally induce BMSCs into an osteoblastic lineage; its effect was transient and it may have been a negative regulator with loading elongation.

The ERK1/2 pathway has been demonstrated to participate in mechano-transduction, turning extracellular mechanical signals into intracellular biological signals (26). Our western blot analysis data revealed that the maximum level of ERK1/2 occurred 30 min after CMS, which confirmed the involvement of the ERK1/2 pathway. U0126 treatment produced a clear downregulation of Runx2, as well as osteogenic-related factors in BMSCs. This was consistent with the findings of previous studies $(27,28)$, showing that Runx 2 can be activated in vivo by ERK1/2 in response to mechanical stress. Since the MAPK family has been shown to be involved in signal transduction that triggers osteoblast differentiation, the present data indicated that CMS could produce a sustained ERK1/2 induction of Runx 2 activation, and this promoted the osteogenic differentiation of BMSCs (Fig. 7).

In summary, we explored the influence of CMS on mesenchymal cell proliferation and osteogenic differentiation. Continuous short-term mechanical stimuli induced the differentiation of BMSCs toward an osteogenic phenotype associated with cessation of proliferation. Runx 2 was shown to be the key factor in osteoblastic differentiation, which could be regulated by ERK1/2. Inhibition of ERK1/2 signaling reduced differentiation of BMSCs into osteoblasts. Increasing evidence for mechanical stimulation as a regulator of osteogenic differentiation, and identification of the relative mechano-transduction pathways are important guides for orthodontic treatment to promote local bone remodeling following orthodontic tooth movement.

\section{Acknowledgements}

This study was supported by the National Natural Science Foundation of China (nos. 30901698, 10972142) and the Science and Technology Commission of Shanghai (no. 08411961600). 


\section{References}

1. Anastasi G, Cordasco G, Matarese G, et al: An immunohistochemical, histological, and electron-microscopic study of the human periodontal ligament during orthodontic treatment. Int $\mathbf{J}$ Mol Med 21: 545-554, 2008.

2. Henneman S, Von den Hoff JW and Maltha JC: Mechanobiology of tooth movement. Eur J Orthod 30: 299-306, 2008.

3. Rath B, Nam J, Knobloch TJ, Lannutti JJ and Agarwal S: Compressive forces induce osteogenic gene expression in calvarial osteoblasts. J Biomech 41: 1095-1103, 2008.

4. Suzuki N, Yoshimura Y, Deyama Y, Suzuki K and Kitagawa Y: Mechanical stress directly suppresses osteoclast differentiation in RAW264.7 cells. Int J Mol Med 21: 291-296, 2008.

5. van Griensven M, Diederichs S, Roeker S, et al: Mechanical strain using 2D and 3D bioreactors induces osteogenesis: implications for bone tissue engineering. Adv Biochem Eng Biotechnol 112: 95-123, 2009.

6. Costessi A, Pines A, D'Andrea P, et al: Extracellular nucleotides activate Runx2 in the osteoblast-like HOBIT cell line: a possible molecular link between mechanical stress and osteoblasts response. Bone 36: 418-432, 2005.

7. Weyts FA, Bosmans B, Niesing R, van Leeuwen JP and Weinans $H$ Mechanical control of human osteoblast apoptosis and proliferation in relation to differentiation. Calcif Tissue Int 72: 505-512, 2003.

8. Byers BA, Pavlath GK, Murphy TJ, Karsenty G and Garcia AJ: Cell-type-dependent upregulation of in vitro mineralization after overexpression of the osteoblast-specific transcription factor Runx2/Cbfal. J Bone Miner Res 17: 1931-1944, 2002.

9. Arnsdorf EJ, Tummala P, Kwon RY and Jacobs CR: Mechanically induced osteogenic differentiation - the role of RhoA, ROCKII and cytoskeletal dynamics. J Cell Sci 122: 546-553, 2009.

10. Hie M and Tsukamoto I: Increased expression of the receptor for activation of NF- $\mathrm{\kappa B}$ and decreased runt-related transcription factor 2 expression in bone of rats with streptozotocin-induced diabetes. Int J Mol Med 26: 611-618, 2010.

11. Lee KS, Kim HJ, Li QL, et al: Runx2 is a common target of transforming growth factor beta1 and bone morphogenetic protein 2 , and cooperation between Runx 2 and Smad5 induces osteoblast-specific gene expression in the pluripotent mesenchymal precursor cell line C2C12. Mol Cell Biol 20: 8783-8792, 2000.

12. Lai CF, Chaudhary L, Fausto A, et al: Erk is essential for growth, differentiation, integrin expression, and cell function in human osteoblastic cells. J Biol Chem 276: 14443-14450, 2001.

13. Simmons CA, Matlis S, Thornton AJ, Chen S, Wang CY and Mooney DJ: Cyclic strain enhances matrix mineralization by adult human mesenchymal stem cells via the extracellular signalregulated kinase (ERK1/2) signaling pathway. J Biomech 36: 1087-1096, 2003.

14. Xiao G, Jiang D, Thomas P, et al: MAPK pathways activate and phosphorylate the osteoblast-specific transcription factor, Cbfa1. J Biol Chem 275: 4453-4459, 2000.
15. Wang FS, Wang CJ, Sheen-Chen SM, Kuo YR, Chen RF and Yang KD: Superoxide mediates shock wave induction of ERK-dependent osteogenic transcription factor (CBFA1) and mesenchymal cell differentiation toward osteoprogenitors. J Biol Chem 277: 10931-10937, 2002.

16. Katz S, Boland R and Santillan G: Modulation of ERK 1/2 and p38 MAPK signaling pathways by ATP in osteoblasts: involvement of mechanical stress-activated calcium influx, PKC and Src activation. Int J Biochem Cell Biol 38: 2082-2091, 2006.

17. Burr DB, Milgrom C, Fyhrie D, et al: In vivo measurement of human tibial strains during vigorous activity. Bone 18: 405-410, 1996.

18. Franceschi RT and Xiao G: Regulation of the osteoblast-specific transcription factor, Runx2: responsiveness to multiple signal transduction pathways. J Cell Biochem 88: 446-454, 2003.

19. Melsen B: Tissue reaction to orthodontic tooth movement - a new paradigm. Eur J Orthod 23: 671-681, 2001.

20. McGuinness NJ, Wilson AN, Jones ML and Middleton J: A stress analysis of the periodontal ligament under various orthodontic loadings. Eur J Orthod 13: 231-242, 1991.

21. Jagodzinski M, Breitbart A, Wehmeier M, et al: Influence of perfusion and cyclic compression on proliferation and differentiation of bone marrow stromal cells in 3-dimensional culture. J Biomech 41: 1885-1891, 2008.

22. Song G, Ju Y, Shen X, Luo Q, Shi Y and Qin J: Mechanical stretch promotes proliferation of rat bone marrow mesenchymal stem cells. Colloids Surf B Biointerfaces 58: 271-277, 2007.

23. Karsenty G: Transcriptional control of skeletogenesis. Annu Rev Genomics Hum Genet 9: 183-196, 2008.

24. Fan X, Rahnert JA, Murphy TC, Nanes MS, Greenfield EM and Rubin J: Response to mechanical strain in an immortalized pre-osteoblast cell is dependent on ERK1/2. J Cell Physiol 207: 454-460, 2006

25. Salingcarnboriboon R, Tsuji K, Komori T, Nakashima K, Ezura Y and Noda M: Runx2 is a target of mechanical unloading to alter osteoblastic activity and bone formation in vivo. Endocrinology 147: 2296-2305, 2006.

26. Kanno T, Takahashi T, Tsujisawa T, Ariyoshi W and Nishihara T: Mechanical stress-mediated Runx2 activation is dependent on Ras/ERK1/2 MAPK signaling in osteoblasts. J Cell Biochem 101: 1266-1277, 2007.

27. Jaiswal RK, Jaiswal N, Bruder SP, Mbalaviele G, Marshak DR and Pittenger MF: Adult human mesenchymal stem cell differentiation to the osteogenic or adipogenic lineage is regulated by mitogen-activated protein kinase. J Biol Chem 275: 9645-9652, 2000.

28. Liedert A, Kaspar D, Blakytny R, Claes L and Ignatius A: Signal transduction pathways involved in mechanotransduction in bone cells. Biochem Biophys Res Commun 349: 1-5, 2006. 